\title{
Transcapillary escape rate of albumin in hypertensive patients with Type 1 (insulin-dependent) diabetes mellitus
}

\author{
K. Nørgaard, T. Jensen and B.Feldt-Rasmussen \\ Steno Diabetes Center, Gentofte, Denmark
}

Summary. Diabetic patients with elevated urinary albumin excretion rate (incipient or clinical nephropathy) also have an increased transcapillary escape rate of albumin. This study was designed to clarify whether this is caused by a general vascular dysfunction or by elevated systemic blood pressure. The systemic blood pressure and the transcapillary escape rate of albumin were measured in the following groups after 4 weeks without antihypertensive treatment: Group 1 eleven healthy control subjects. Group 2 - ten Type 1 (insulin-dependent) diabetic patients with incipient nephropathy (urinary albumin excretion rate: $30-300 \mathrm{mg} / 24 \mathrm{~h}$ ) and normal blood pressure. Group 3 - eleven non-diabetic patients with essential hypertension. Group 4 - nine Type 1 diabetic patients with hypertension but normal urinary albumin excretion ( $<30 \mathrm{mg} / 24 \mathrm{~h}$ ). Group 5 - eleven Type 1 diabetic patients with nephropathy (urinary albumin excretion rate $>300 \mathrm{mg} / 24 \mathrm{~h}$ ) and hypertension. Systolic and diastolic blood pressure were similar in the three hypertensive groups: group 3, $148 \pm 8 / 95 \pm 6$; group 4, $150 \pm 12 / 94 \pm 8$ and group $5 ; 152 \pm 12 / 92 \pm 7 \mathrm{~mm} \mathrm{Hg}$, but significantly elevated $(p<0.001)$ compared to control group $1,117 \pm 12 / 74 \pm 9$ and group 2, $128 \pm 7 / 82 \pm 4 \mathrm{mmHg}$. The transcapillary escape rate of albumin was similar in the control subjects $(5.2 \pm 2.7 \%)$ and the subjects in the normoalbuminuric groups 3 and $4(6.2 \pm 1.9$ and $5.1 \pm 1.4 \%$, respectively) and significantly lower $(p<0.001)$ than in patients with elevated urinary albumin excretion without or with hypertension group $2,10.1 \pm 2.8$ and group $5,11.4 \pm 5.7 \%$. The increased transcapillary escape rate of albumin in patients with elevated urinary albumin excretion is unrelated to moderate systemic hypertension and may therefore be caused by alterations in the properties of the capillary walls.

Key words: Type 1 (insulin-dependent) diabetes mellitus, mild hypertension, diabetic nephropathy, albumin leakage.
The role of hypertension in the development of diabetic nephropathy is still debated. The question is whether hypertension is an initiating factor of diabetic nephropathy causing albuminuria or whether hypertension is only secondary to other factors which lead to the increased transglomerular albumin sieving of nephropathy. We have suggested that the cause of the elevated transglomerular passage of albumin is genetically determined alterations in the composition of the extracellular matrix [1]. These alterations may also be present in extra-renal capillaries and thus, albuminuria reflects more generalized vascular damage. This hypothesis was based on studies of the transcapillary escape rate of albumin $\left(T R_{a l b}\right)$. TER $R_{a b b}$ is a measure of the unidirectional microvascular flux of plasma albumin in renal and extra-renal tissues [2]. It has been interpreted as a marker of general vascular dysfunction [3]. In our study of long-term diabetic patients with no signs of late diabetic complications (i. e. normal urinary albumin excretion) we observed a normal TER $\mathrm{R}_{\mathrm{alb}}$ [4]. In contrast, an elevated TER $\mathrm{alb}_{\mathrm{b}}$ was seen in patients with normal blood pressure but slightly elevated urinary albumin excretion (UAE) rate (incipient nephropathy). The TER $\mathrm{Tab}_{\mathrm{ab}}$ in these normotensive patients was elevated to the same degree as in patients with clinical nephropathy.

The present study was designed to further clarify the roles of vascular dysfunction and elevated systemic blood pressure on the TER $\mathrm{Talb}_{\mathrm{b}}$ in Type 1 (insulin-dependent)

Table 1. Main inclusion criteria for the five groups of subjects studied. Presence or absence of each condition is indicated by + and -

\begin{tabular}{llll}
\hline & Type 1 diabetes & Hypertension & Albuminuria \\
\hline Group 1 & - & - & - \\
Group 2 & + & - & + \\
Group 3 & - & + & $-1+$ \\
Group 4 & + & + & - \\
Group 5 & + & + & + \\
\hline
\end{tabular}


diabetic patients. The aim was to study, in the presence or absence of microalbuminuria, the impact of moderately increased blood pressure on the transport of albumin over the total microvascular bed in diabetic patients, i. e. on the TER alb.

\section{Subjects and methods}

\section{Patients}

Five groups of subjects were studied. The subjects were aged 18-50 years (Table 1) and none were obese i.e. body mass in$\operatorname{dex} \leq 28 \mathrm{~kg} / \mathrm{m}^{2}$. Group 1: eleven healthy control subjects with no family history of diabetes or hypertension. Group 2: ten Type 1 diabetic patients with incipient nephropathy i.e. UAE persistently in the range $30-300 \mathrm{mg} / 24 \mathrm{~h}$. These patients were randomly selected from patients with normal blood pressure i.e. systolic pressure below $140 \mathrm{~mm} \mathrm{Hg}$ and diastolic pressure below $90 \mathrm{~mm} \mathrm{Hg}$, according to the WHO criteria for borderline hypertension. Group 3: eleven non-diabetic patients diagnosed with essential hypertension as secondary hypertension was excluded using standard procedures. Inclusions were made irrespective of their UAE rate. After 4 weeks without antihypertensive treatment patients should have a systolic blood pressure $\geq 140 \mathrm{mmHg}$ or a diastolic blood pressure $\geq 90 \mathrm{~mm} \mathrm{Hg}$ or both. Group 4: nine Type 1 diabetic patients with elevated blood pressure after 4 weeks without antihypertensive treatment (as defined for group 3). UAE measured annually in the clinic had never been above $30 \mathrm{mg} / 24 \mathrm{~h}$ either before or after antihypertensive treatment (if any) was initiated. Fulfilling these criteria, we defined these patients as having essential hypertension coexistent with Type 1 diabetes as previously described [5]. Group 5: eleven Type 1 diabetic patients with clinical nephropathy, i.e. $\mathrm{UAE} \geq 300 \mathrm{mg} / 24 \mathrm{~h}$ in at least two out of three urine collections and other renal diseases excluded. All had hypertension, as defined for groups 3 and 4 , and serum creatinine was normal $(<100 \mu \mathrm{mol} / /)$.

In all the patients with Type 1 diabetes (those in groups 2,4 and 5) the onset of diabetes had been prior to the age of 31 years, and these patients were randomly selected from patients fulfilling the criteria for each group who were attending Steno Diabetes Center. The patients in group 3 were attending the Hypertension Clinic at Bispebjerg Hospital, Copenhagen and were also randomly selected. In order to avoid development of severe hypertension during 4 weeks without antihypertensive treatment, the patients in the three hypertensive groups (groups 3, 4 and 5) were selected from those patients whose blood pressure was relatively well-controlled ( $\leq 170 / 100)$ by taking two different antihypertensive drugs or less.

Informed consent for participation was obtained, and the study was approved by the Regional Scientific Ethics Committee.

\section{Study design and methods}

All patients were studied in our laboratory after having discontinued antihypertensive treatment for at least 4 weeks (if on treatment). Blood pressure was measured every week. If systolic pressure exeeded $200 \mathrm{~mm} \mathrm{Hg}$ and/or diastolic pressure exeeded $110 \mathrm{~mm} \mathrm{Hg}$ or in case of any discomfort of the patients ( $n=1$ from group 4 and $n=5$ from group 5 ), the patients were withdrawn from the study and antihypertensive treatment instituted again. Numbers of patients using the following antihypertensive drugs: none/diuretics/betablockers/angiotensin converting enzymes/calcium channel blockers prior to the study were, for group $3: 7 / 3 / 0 / 2 / 1$, for group $4: 0 / 5 / 1 / 2 / 1$, and for group 5:0/5/2/4/3. In the last week before the study the patients in groups 2-5 collected two 24-h urine samples for UAE rate determination. All subjects were studied after an overnight fast, the diabetic patients omitting their morning insulin. A cannula was inserted in to the antecubital vein in each arm and blood samples were collected after 30 -min rest in the supine position. Blood pressure was measured twice with a random zero sphygmomanometer (Hawksley, West Sussex, UK) and expressed as the mean value. The TER is the fraction of the intravascular albumin mass leaving the vascular bed per hour $(\% / h)$. TER alb $_{\text {bas }}$ determined after an intravenous injection of $40 \mathrm{kBq}{ }^{125} \mathrm{I}$-labelled human serum albumin. The radioactivity of plasma sampled at $10,20,30,40,50,60$ min after the injection was used for calculation. TER $_{\mathrm{atb}}$ is the rate constant of the practically monoexponential decrease in plasma radioactivity over the first $60 \mathrm{~min}$ after the injection of the tracer, and calculated by the least squares method [6]. The haematocrit was measured in each sample and the plasma radioactivity was expressed according to the small change in plasma volume during the sampling period. The TER $_{\mathrm{alb}}$ measurement was accepted only if the correlation coefficient between the time points for blood sampling and the corresponding values of in (plasma radioactivity) exceeded 0.85 . Because of this criteria three patients were excluded from the study. The degree of diabetic retinopathy was scored after direct ophthalmoscopy through dilated pupils as none, background retinopathy, proliferative retinopathy or blindness.

\section{Laboratory measurements}

Haemoglobin $\mathrm{A}_{1 \mathrm{c}}$ was measured by HPLC (Bio Rad DIAMAT, Richmond, Calif., USA) (normal range 4.3-6.2\%). Blood glucose was measured by means of Hypocount B (Hypoguard LD, Woodbridge, Suffolk, UK). Serum creatinine was measured by a reaction rate kinetic principle eliminating pseudo-creatinines [7]. Urinary albumin concentration was determined using an ELISA-assay and expressed as median of three 24 -h collections [8].

\section{Statistical analysis}

Results are given as mean $\pm \mathrm{SD}$ for the normally distributed data. UAE rates are given as median and range. Significant group differences were detected by Duncan's multiple range test. The test was performed for UAE after logarithmic transformation of the data. $\mathrm{Li}$ near regression analysis was used to detect any correlations between TER $_{\mathrm{alb}}$ and blood pressure. Comparison of retinopathy in the groups was performed using chi-square test. A $p$-value (two-tailed) below 0.05 was considered significant.

\section{Results}

Clinical data are given in Table 2. The five groups were matched for age and gender distribution. Diabetes duration in the three diabetic groups was similar. The diabetic groups were treated with a comparable daily dose of insulin. The glycaemic control $\left(\mathrm{HbA}_{1 c}\right)$ differed between the three diabetic groups. Group $2: 9.2 \pm 1.0 \%$ was significantly higher than group 4: $7.8 \pm 0.8 \%$ and group 5: $8.2 \pm 1.1 \%(p<0.001)$. The actual blood glucose on the study day was similar in all the diabetic groups. The degree of retinopathy in the diabetic groups was scored as none/background retinopathy/proliferative retinopathy/blindness. Patients with coexistence of essential hypertension had significantly less retinal changes than the albuminuric groups; group $4: 6 / 3 / 0 / 0$ vs group $2: 2 / 5 / 1 / 2$ $(p<0.05)$ and vs group 5:0/3/6/2 $(p<0.05)$. Both systolic and diastolic blood pressure were comparable in the three hypertensive groups (group 3: systolic $148 \pm 8$, diastolic $95 \pm 6$; group 4: systolic $150 \pm 12$, diastolic $94 \pm 8$; and group 5: systolic $152 \pm 12 \mathrm{~mm} \mathrm{Hg}$, diastolic $92 \pm 7 \mathrm{~mm} \mathrm{Hg}$ ) and elevated as compared to the control subjects $(117 \pm 12 / 74 \pm 9 \mathrm{~mm} \mathrm{Hg}, p<0.001)$ and the normotensive patients with microalbuminuria $(128 \pm 7 / 82 \pm 4, p<0.001)$ (Fig. 1). Normotensive diabetic patients with incipient nephropathy had slightly elevated blood pressure compared 
Table 2. Clinical data of control subjects (group 1), Type 1 diabetic patients with microalbuminuria and normotension (group 2), essential hypertensive non-diabetic patients (group 3), essential hyper- tensive Type 1 diabetic patients (group 4) and Type 1 diabetic patients with nephropathy and hypertension (group 5)

\begin{tabular}{llcccc}
\hline & Group 1 & Group 2 & Group 3 & Group 4 & Group 5 \\
\hline Sex (male/female) & $(6 / 5)$ & $(9 / 1)$ & $(8 / 3)$ & $(6 / 3)$ & $(10 / 1)$ \\
Age (years) & $37.9 \pm 7.7$ & $37.9 \pm 8.3$ & $37.1 \pm 8.0$ & $40.4 \pm 5.6$ & $40.8 \pm 7.3$ \\
Diabetes duration (years) & - & $24.8 \pm 7.6$ & - & $18.0 \pm 11.1$ & $24.5 \pm 6.7$ \\
Insulin dose $\left(\mathrm{IU} \cdot \mathrm{kg}^{-1} \cdot 24 \mathrm{~h}^{-1}\right)$ & - & $0.58 \pm 0.18$ & - & $0.53 \pm 0.12$ & $0.63 \pm 0.08$ \\
$\mathrm{HbA}_{\mathrm{ic}}(\%)^{\mathrm{b}}$ & $4.9 \pm 0.3^{\mathrm{c}}$ & $9.2 \pm 1.0^{\mathrm{d}}$ & $4.9 \pm 0.4^{\mathrm{c}}$ & $7.8 \pm 0.8$ & $8.2 \pm 1.1$ \\
Blood glucose $(\mathrm{mmol} / \mathrm{l})^{\mathrm{b}}$ & $4.3 \pm 1.3^{\mathrm{c}}$ & $12.2 \pm 2.5$ & $5.2 \pm 0.7^{\mathrm{c}}$ & $13.1 \pm 3.3$ & $11.3 \pm 4.8$ \\
$\mathrm{UAE}(\mathrm{mg} / 24 \mathrm{~h})$ & $8(4-21)$ & $102(30-293)$ & $19(3-55)$ & $7(3-25)$ & $864(326-3837)$ \\
S-creatinine $(\mu \mathrm{mol} / \mathrm{l})^{\mathrm{a}}$ & $84 \pm 11$ & $81 \pm 8$ & $78 \pm 8$ & $72 \pm 8^{\mathrm{c}}$ & $86 \pm 11$ \\
\hline
\end{tabular}

Statistical significant group difference (Duncan's test): ${ }^{\mathrm{a}} p<0.05 ;{ }^{\mathrm{b}} p<0.001$. Statistical significant difference between the groups: ${ }^{\mathrm{c}}$ vs group 2,4 and $5 ;{ }^{\mathrm{d}}$ vs group 3 and $4 ;{ }^{\mathrm{e}}$ vs group 1 and 5.

UAE, urinary albumin excretion

to the control subjects. A statistically significant group difference was documented for $\operatorname{TER}_{\text {alb }}(p<0.001)$. The TER alb was similar in control subjects (group 1: $5.2 \pm 2.7 \%$ ), non-diabetic subjects with essential hypertension (group 3:6.2 $\pm 1.9 \%$ ) and Type 1 diabetic patients with essential hypertension (group 4:5.1 $\pm 1.4 \%$ ), and significant lower than in diabetic patients with clinical nephropathy (group 5: $11.4 \pm 5.7 \%$ ) and microalbuminuria (group 2: $10.1 \pm 2.8 \%$ ) (Fig. 2). No significant correlation was found between either systolic or diastolic blood pressure and TER $\mathrm{R}_{\text {alb }}$ in any of the groups. Neither was there any significant correlation between blood pressure and TER $_{\mathrm{alb}}$ when testing the albuminuric diabetic patients (groups 2 and $5, n=21$ ).

\section{Discussion}

This study has shown that TER $_{\text {alb }}$ is elevated in Type 1 diabetic patients with elevated UAE regardless of whether the blood pressure was elevated, and that TER $\mathrm{R}_{\mathrm{alb}}$ is normal in moderately hypertensive diabetic patients, with a normal UAE, as well as in non-diabetic patients with essential hypertension.
The elevated TER $\mathrm{R}_{\mathrm{ab}}$ in albuminuric patients does not seem to be explained by increased urinary albumin loss. Assuming a plasma volume of $3000 \mathrm{ml}$ and a plasma concentration of albumin $40 \mathrm{~g} / \mathrm{l}$, an increase in TER $\mathrm{alb}_{\mathrm{ab}}$ from $5 \%$ to $10 \%$, as reported in the present study, represents an increased albumin leakage of $6 \mathrm{~g} / \mathrm{h}$. None of the albuminuric diabetic patients had a UAE higher than $160 \mathrm{mg} / \mathrm{h}$, and $\mathrm{TER}_{\mathrm{alb}}$ was increased to the same level in patients with incipient and clinical nephropathy despite a 10 -fold difference in UAE between the two groups.

In the present study, the two groups of hypertensive diabetic patients (group 4 and group 5) had the same moderate degree of hypertension, the same diabetes duration and similar blood glucose values during the measurement of TER $\mathrm{alb}_{\mathrm{b}}$ but the TER $\mathrm{Tab}_{\mathrm{ab}}$ was elevated only in the presence of an excess loss of albumin in the urine (group 5). This suggests that generalised endothelial cell dysfunction is responsible for the increased TER $\mathrm{R}_{\mathrm{abb}}$ in this group of patients, as also discussed below. Generalised endothelial dysfunction is evident in diabetic patients with nephropathy $[3,9,10]$. A previous study by our group [11] also gave support to the concept that the level of the $\mathrm{TER}_{\mathrm{alb}}$ should be related to generalized vascular dysfunc-

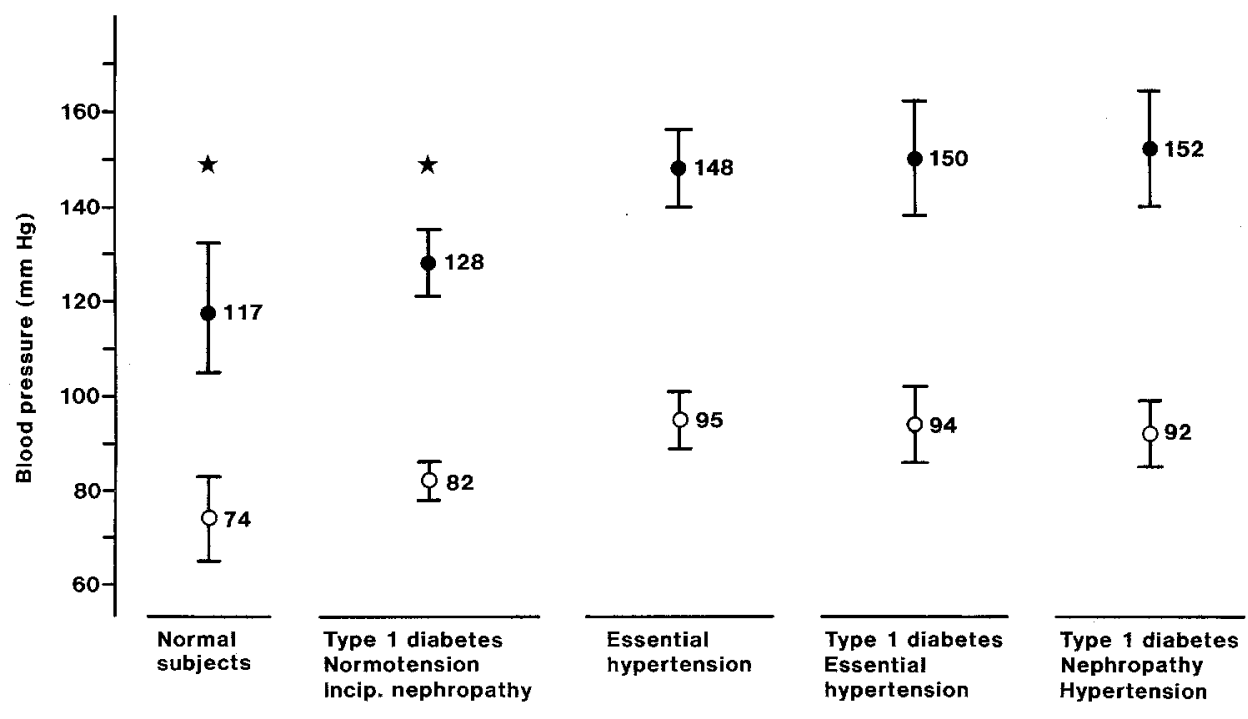

Fig. 1. Blood pressure in the five groups studied. Mean \pm 1 SD are given. : Systolic blood pressure, $O$ : diastolic blood pressure. Statistical significant group difference $(p<0.001),(*)$ : vs all other groups 


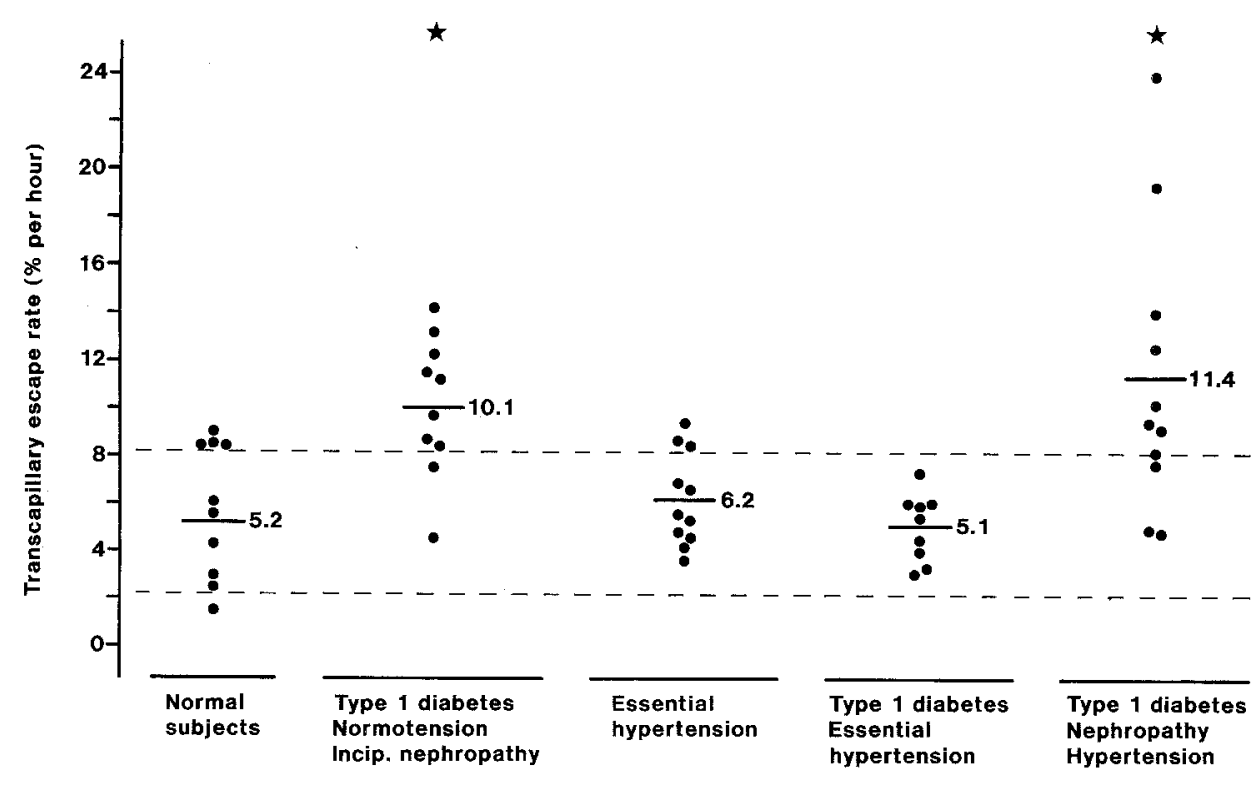

Fig.2. Transcapillary escape rate of albumin in the groups studied. Statistical significant group difference $(p<0.001),(*)$ : vs groups 1,3 and 4 . Dotted lines indicate the mean $\pm 2 \mathrm{SD}$ in Type 1 diabetic patients with normoalbuminuria and normotension (values from a previous study in our laboratory [4]) tion. In this study, the TER $\mathrm{R}_{\mathrm{ab}}$ was lowered in diabetic patients with early nephropathy receiving dietary cod-liver oil supply for 8 weeks. The change in $T^{2} R_{a b b}$ was not correlated to the small reduction seen in blood pressure. Furthermore, in a previous study in normotensive albuminuric patients, the TER ${ }_{\text {alb }}$ remained stable in spite of a reduction of $8 \mathrm{mmHg}$ systolic and $5 \mathrm{mmHg}$ diastolic blood pressure [12].

In previous studies different factors were found to be of importance for the level of the TER $\mathrm{R}_{\mathrm{alb}}$ in Type 1 diabetic patients. Poor glycaemic control has been demonstrated to increase vascular permeability in short-term diabetic patients [13]. In a study comprising some Type 1 but mostly Type 2 (non-insulin-dependent) diabetic patients without complications, O'Hare and co-workers [14] were able to reduce TER $_{\text {alb }}$ from $8.8 \%$ to $6.3 \%$ by improving glycaemic control for 3 weeks. However, in patients with microalbuminuria, the glycaemic control did not seem to contribute to the level of TER $\mathrm{alb}_{\text {alb }}$ [4]. In the present study we found that group 4 of diabetic patients with essential hypertension and normal TER $\mathrm{alb}_{\mathrm{b}}$ had significantly lower $\mathrm{HbA}_{1 c}$ than microalbuminuric normotensive patients. The actual blood glucose was however similar between the groups and $\mathrm{Hb} \mathrm{A}_{1 \mathrm{c}}$ was similar between groups 4 and 5 . Furthermore, there was no significant correlation between $\mathrm{HbA}_{\mathrm{ic}}$ and $\mathrm{TER}_{\mathrm{alb}}$. Therefore, the observed differences in TER $\mathrm{R}_{\mathrm{alb}}$ are not likely to be explained by differences in glycaemic control.

It is well known that TER $\mathrm{alb}_{\mathrm{ab}}$ is elevated in essential hypertensive patients, with very high blood pressure levels in whom a correlation between TER $\mathrm{R}_{\mathrm{alb}}$ and blood pressure has been described [6]. We found a normal TER $_{\mathrm{alb}}$ in non-diabetic patients with essential hypertension possibly because we studied only patients with moderate hypertension. Thus, in a previous study from Parving and Gyntelberg [6] the TER abb $_{\text {ab normal up to a }}$ level of diastolic pressure of $120 \mathrm{~mm} \mathrm{Hg}$ and none of the hypertensive patients in our study exceeded that level. It is relevant to include only patients with low range hypertension when studying the mechanisms initiating diabetic ne- phropathy, because the blood pressure in patients with incipient nephropathy is only slightly elevated $[15,16]$. O'Hare et al. [14] stated that hypertension independently increases $T_{E R} R_{a b b}$ in diabetic patients without complications. In their study the normotensive and hypertensive patients were not matched as in the present study, specifically with respect to the type of diabetes, age and weight. Their results are in contrast to those presented here where we found that moderate hypertension, in Type 1 diabetic patients free of severe long-term complications, does not influence the TER $\mathrm{alb}_{\mathrm{b}}$. The TER $\mathrm{Talb}_{\mathrm{b}}$ was elevated only in the presence of elevated albuminuria and unrelated to the presence of hypertension.

Autoregulation of blood flow is the ability to maintain almost constant blood flow in the microcirculation during changes in the arterial blood pressure. Theoretically, an impaired autoregulation could affect the level of TER ${ }_{\text {alb }}$. We did not evaluate the autoregulatory capacity of the patients. It is, however, unlikely that the observed association of an elevated TER alb $_{\text {and }}$ an increased UAE could be explained by loss of autoregulatory capacity, as quite dramatic differences in autoregulation should be present to explain an increase in $\mathrm{TER}_{\mathrm{alb}}$ in patients with a mean blood pressure an average of $16 \mathrm{~mm} \mathrm{Hg}$ below the level of patients with normal TER $\mathrm{R}_{\mathrm{abb}}$ (group 2 vs group 4).

In conclusion, increased TER $\mathrm{R}_{\mathrm{ab}}$ in patients with elevated UAE is unrelated to moderate systemic hypertension and seems rather to be caused by alterations in the properties of the capillary walls.

Acknowledgements. This study was supported by a grant from the Danish Diabetes Association (Diabetesforeningen). We thank Dr. E.Rasmussen, Bispebjerg Hospital, Copenhagen, for carefully selecting the non-diabetic patients with essential hypertension and Ms. H.Foght for her skillful technical assistance.

\section{References}

1. Deckert T, Feldt-Rasmussen B, Borch-Johnsen K, Jensen T, Kofoed-Enevoldsen A (1989) Albuminuria reflects widespread vascular damage. The Steno hypothesis. Diabetologia 32:219-226 
2. Parving H-H (1991) Impact of blood pressure and antihypertensive treatment on incipient and overt nephropathy, retinopathy, and endothelial permeability in diabetes mellitus. Diabetes Care 14:260-269

3. Jensen T, Bjerre-Knudsen J, Feldt-Rasmussen B, Deckert T (1989) Features of endothelial dysfunction in early diabetic nephropathy. Lancet I: 461-463

4. Feldt-Rasmussen B (1986) Increased transcapillary escape rate of albumin in type 1 (insulin-dependent) diabetic patients with microalbuminuria. Diabetologia 29: 282-286

5. Nørgaard K, Feldt-Rasmussen B, Borch-Johnsen K, Sælan H, Deckert $T$ (1990) Prevalence of hypertension in type 1 (insulindependent) diabetes mellitus. Diabetologia 33: 407--410

6. Parving H-H, Gyntelberg F (1973) Transcapillary escape rate of albumin and plasma volume in essential hypertension. Circ Res 32: $643-651$

7. Larsen K (1972) Creatinine assay by a reaction-kinetic principle. Clin Chem Acta 41:209-217

8. Feldt-Rasmussen B, Dinesen B, Deckert M (1985) Enzyme immuno assay- an improved determination of urinary albumin in diabetics with incipient nephropathy. Scand J Clin Lab Invest 45: $539-544$

9. Jensen T (1989) Increased plasma levels of von Willebrand factor in insulin dependent diabetic patients with incipient nephropathy. Br Med J 298: 27-28

10. Lieberman J, Sastre A (1980) Serum angiotensin-converting enzyme: elevations in diabetes mellitus. Ann Intern Med 93: 825 826

11. Jensen T, Stender S, Goldstein K, Hølmer G, Deckert T (1989) Partial normalization by dietary cod-liver oil of increased micro- vascular albumin leakage in patients with insulin-dependent diabetes and albuminuria. N Eng J Med 321: 1572-1577

12. Nørgaard K, Jensen T, Feldt-Rasmussen B (1992) Effects of Isradipine in type 1 (insulin-dependent) diabetic patients with albuminuria and normal blood pressure. J Human Hypertension 6:145-150

13. Parving H-H, Noer J, Deckert T et al. (1976) The effect of metabolic regulation on microvascular permeability to small and large molecules in short-term juvenile diabetics. Diabetologia 12: 161166

14. O'Hare JA, Ferriss JB, Twomey B, O'Sullivan DJ (1983) Poor metabolic control, hypertension and microangiopathy independently increase the transcapillary escape rate of albumin in diabetes. Diabetologia 25:260-263

15. Feldt-Rasmussen B, Borch-Johnsen K, Mathiesen ER (1985) Hypertension in diabetes as related to nephropathy. Hypertension 7 [Suppl 2]: II-18-II-20

16. Mathiesen ER, Rønn B, Jensen T, Storm B, Deckert T (1990) Relationship between blood pressure and albumin excretion in development of microalbuminuria. Diabetes 39: 245-249

Received: 6 July 1992

and in revised form: 10 September 1992

Dr. K. Nørgaard

Steno Diabetes Center

DK-2820 Gentofte

Denmark 